$\eta_{\text {LA-6420-MS }} \quad \phi-223$

Informal Report

UC.21

Reporting Date: June 1976

Issued: July 1976

\title{
Dependence of Laser-Driven Compression Efficiency on Wavelength
}

by

R. L. McCrory

R. L. Morse

of the University of California

LOS ALAMOS, NEW MEXICO 87545

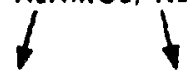

An Alfirmalive Action/Equal Opportunity Employer 
This work supported by the US Energy Research and Development Administration's Division of Laser Fusion and Division of Military Application.

Printed in the United States of America. Available from

National Technical Information Service

U.S. Department of Cornmerce

5285 Port Royal Road

Springfield, VA 22161

Price: Printed Copy \$3.50 Microfiche \$2.25

Thin report was prepared an en apcount of work uponeored

by the United Sinles Governmenl. Nether tha United Statea

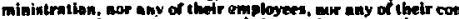

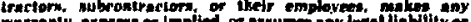

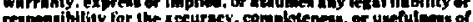

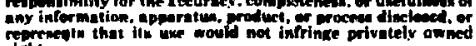

rethis. 
DEPENDENCE OF IASER-DRIVEN COMPRESSION

EFEICIENCY ON WAVELENGTH

by

R. L. McCrory and R. L. Morse

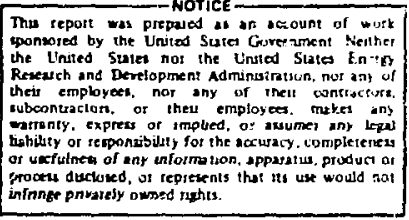

ABSTRACT

Numerical simulacions of laser-driven Implesions combined with previously derived scaling laws, both based on classical thermal conduction without any flux limiting, indicate that the absorbed energy required to cause a given implosion, and, therefore, the anticipated nuclear yleld ratio should scale approximately inversely with wavelength in the visible and near infrared.

\section{INTRODUCTION}

Extensive numerical simulations indicate the possibility of achieving energy breakeven or better from laser-driven spherical implosion of pellets containing thermunuclear fuston fuel. ${ }^{1}$ Implosion is driven by ablation of malirial from the surface of a pellet by the absorbed laser energy. Th1s energy is transported by electron thermal conduction from the surface of critical density, $\rho_{c}$, where it is absorbed from the incident laser light, to tha surface of the pellet core, or ablation surface. (Recall that the ablation surface essentially separates the blowoff from the dense pellet core which is being compressed.) For visible or infrared wavelengths, $\lambda, \rho_{c}$ is much less than the density of the pellet core and occurs in th: 10 density blowoff. For the $\mathrm{Nd}$ laser from which $\lambda$, the wavelength, is 1.06 .in, the critical electron density is $\mathrm{n}_{c}=10^{21} \mathrm{~cm}^{-3}$, and in the compressed core, which is at solid densities or higher, $n_{c} \geq 10^{23} \mathrm{~cm}^{-3}$. In Ref. 2 it is shown by a stationary flow model of the thermal conduction and ablation process that, among other things, the absorbed laser power, $w$, required to provide a given ablation pressure and material ablation rate increases with decreasing $\rho_{c}$. This result is a consequence of the relatively larger radius of the critical surface when $\rho_{c}$ is made relatively smaller, which restits in a louger region through which the absorbed power must be conducted before reaching the ablation surface. In particular, Ref. 2 finds tiat, to a good approximation, the required power scales as $W \sim \rho_{c}^{-\frac{1}{2}}$, and, therefore, since $\rho_{c} \sim \lambda^{-2}$, that $w \sim \lambda$. The 1ndicated scaling wouli be very important for laser fusion research and development because a factor of $\lambda$ woü multiply $^{-}$ pellet yiald ratios, and there are differences of an order of magnitude or more in the wavelengths of the different lasers being considered for this purpose. We show here that this scaling is applicable to a range of values of $\lambda$ of interest in laser fusion research. The short wavelength end of the range is simply determined by the requirement that $f_{c}$ be less than the initial solld density of a pellet. Reascns can be given for keeping $\rho_{c}$ at least a few times smaller than Initial solid density. This requires that the critical electron density be less than about $10^{23} \mathrm{~cm}^{-3}$ or that $\lambda \geq 10^{-1}$ $\mu \mathrm{m}$. The large $\lambda$ und of the range of applicability must then be somewhat larger than $\lambda=10^{-1} \mu \mathrm{m}$ in order for the $W \sim \lambda$ scaling to be important. This end of the range is determined by the validity of the stationary flow model and occurs, as discussed in Ref. 2, where $\rho_{c}$ is so small that the $t$ ime required for material to flow from the ablation surface to the critical surface is no longer small. 
compared to the implostcn time. The following timedependent numertcal simulation parameter study of iaser-driven implosions Indicates thi existence of a range of approximate validity of the $W \sim \lambda$ scaling, and the approximate location of the large $\lambda$ end of the range.

\section{NUMERICAL PARAMETER STUDY}

The simulation paramecer study was done with a one-ulmensional, spherical, Lagranglan hydrodynamica and heat flow code. In order to allow direct comparison to the model of Ref. 2 and to permit scaling the results to a wide range of physical cases, we have used an ideal gas equation of state, in conjunction with a one-temperature model (for tons and electrons) and, in the electron thermal conduction, $\ln A=$ const. $=5$. The target is a $10^{2} \mu \mathrm{m}$ radius sphere of $Z=1, A=2.5$ material of initial density $1 \mathrm{gm} / \mathrm{cm}^{3}$. The simple scaling laws of tydrodynamicg and one cemperature heat flow readily transform this case into one of higher or lower initial density and higher $Z$ withoul changing the calculated efficiencies for a given pulse shape. Constant power step funccion p:ilses of a range of intensities are used. The laser pulse is never shut off in these calculations, but any additional energy supplied to the pellet after peak compression need not be considered. Both more elaborate target and pulse shapes could have been used which would give much larger values $\rho R$ (see below). However, since the object of this study is only to show the dependence of the energy transfer to the imploded part of the pellet on $P_{c}$, a different, more complex, chcice of target and pulse shape would, if anything, reduce the generalfty of the results.

Figure la shows the peak value of $\int_{0}^{\infty} \mathrm{dr} \rho$, called $O R$, a common figure of merit for spherical compression (see Ref. 1), for a range of pulse powers and for the critical densities $\rho_{c}=10^{-1}, 10^{-2}$, and $10^{-3}$. Power is given in units of atts $/ \mathrm{cm}^{2}$ at the inftial pellet radius. The sligint rise of $\rho R$ for irradiances above $10^{16} \mathrm{w} / \mathrm{cm}^{2}$, followed by a drop to the Initial time value, occurs shen the incldent power becomes so large that the Inward-moving thermal wave front begins to keep up with or outruns the converging shock, viz., the so-called "burn through" 11mit. Note that at lower power $\rho R \simeq 0.041$ s wat one expects from a one-shock implosion, and the effect of changing $P_{c}$ and $W$ is just to alter the ahsck timing. Figure Ib shows the internal energy st the tine of peak $O R$ in that part of the pellet mass responsible for the innermost $80 \%$ of $p R$. The use of this $80 \%$ prescription has been found to be a generally reliabie procedure for Identifying parameters of the compressed core, at least for the kind of aimple carget and pulse considered here. Examintion of further detalis of the simulation results show, as would be expected, that in the lower power range, where $\rho R$ vs $W I E$ nearly flat, cases with different $\rho_{c}$, which have the same incernal energy in the core, are quite simila: in all respects Inside of the sbiation surface. Two sets of such cases, three at 0.33 joules of internal eneisy and different $\rho_{c}$, and three at 8.6 joules, are c1rclei on F18. $1 b$, and the same cases are circled on Fig. 1c which 18 a plot of efficiency, $\eta$, of transfer of absorbed energy, $W$, Into internal energy. That is, $\eta$ is the ratio of the internal energy plotted in Fig. 1c to the total energy absorbed lit the pellet up to the time of peak $\rho R$. At 0.33 foules, the riatio of the values of $n$ at $\rho_{c}=10^{-2}$ and $10^{-1}$ (rf sall that the inftial density is $1 \mathrm{gm} / \mathrm{cm}^{3}$ so that these values of $\rho_{c}$ are numerically the ratio of $\rho_{c}$ to the pellet density) is about 2.5 or almost the ratlo of wavelengths, $\sqrt{10}$, while the ratio of $\eta^{\prime} s$ at $10^{-3}$ and $10^{-2}$, approximately 1.7, is significantly lower. At 8.6 joules, where the mass ablation rate is larger and the stationary ablation model is not expected to be as good, the effletency is better but the respective ratios; 2.2 and 1.2 , are $a$ bit omaller. If the Initial density of $1 \mathrm{gm} / \mathrm{cm}^{3}$ is scaled up to typical outside surface target material densities of about $2 \mathrm{gm} / \mathrm{cm}^{3}$, then the wavelength corresponding to our highest $\rho_{c}$, which becomes 0.2 $\mathrm{gm} / \mathrm{cm}^{3}, 1 \mathrm{~s}$ about $0.15 \mathrm{\mu m}$, and that corresponding to the smallest is $1.5 \mu \mathrm{m}$. The gimulations show that the acaling is weakening considerably at the longer wavelength, which appears to be approximately the large $\lambda$ end of the range of validity of the $w \sim \lambda$ scaling. Different pulse shapes and target configuratione could, by shanging the time scales of implosions and transients in the ablation fiow, change the large $\lambda$ end of the range by perheps a factor of two, but would probably not extend the range as far as $10 \mathrm{\mu m}$. We also expect that those pulse shapes which extend the range w11l give a 
scallng of $W$ with $\lambda$ which $1 \mathrm{~s}$ closer co $H \sim \lambda$ than was seen in our parameter study with step function pulses.

\section{CONCLUSIONS}

Our conclusion frotn these preliminary calculations is that the efflciency with which absorbed laser energy causes a given spherical implosion in medium to low 2 materials should increase by a factor of between three and five if the laser wavelength is decreased irom infrared wavelengths between 1 and $10 \mathrm{~lm}$ to the blue or near ultraviulet A small additional improvement might be gained with some targets by going down into the vacuum ultraviolst, below about $0.2 \mu \mathrm{m}$, but at the expense of some increase in experimental difficulty. These
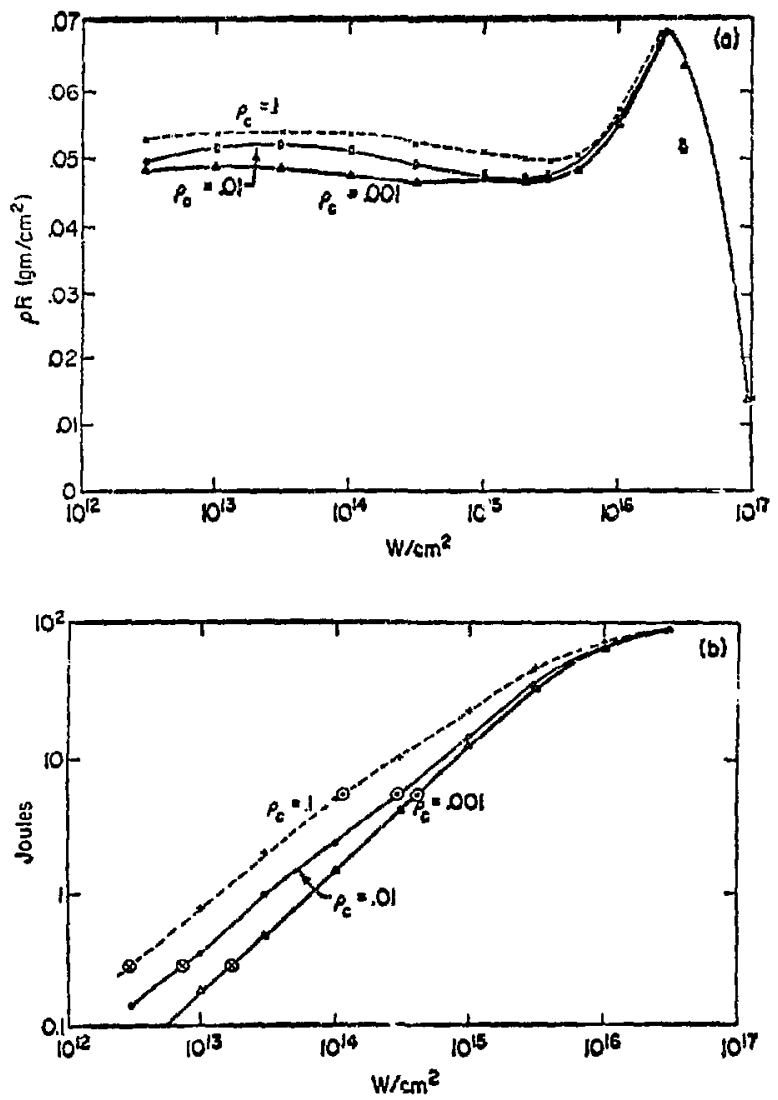

calculations, which consider only classfcal thermal conductivity, indicate that the further Ioss in efficiency from going io wavelengths as long as $\mathrm{J} 0$ um and longer should be smal1. This effect should not be confused with nonclassical thermal ilux liwiting, which may Intraduce some inefficiency at wavelengths as short as $1 \mathrm{um}^{3}$

\section{REPERENCES}

1. T. P. Hughes, Plasmas and Laser Light (John Wiley and Sons, Inc, NY, 1975).

2. S. J. Gitomer, R. I. Morse, and B. S. Newberger, "Structure and Scaling Laws of Laser Driven Implosions," Los Alamos Scientific Laboratory Report LA-6079-MS (to be published in Phys. Flulds).

3. R. C. Malone, R. L. McCrory, and R. L. Morse, Phys. Rev. Lett. 34, 721 (1975).

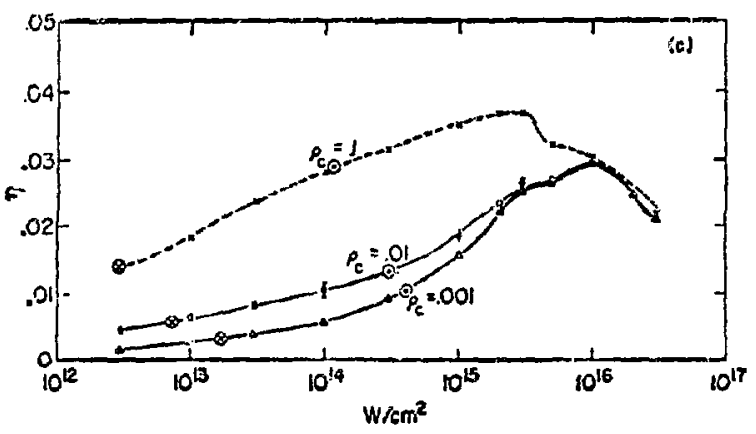

Fig. 1. Results of a numerical simulation param meter study of laser-driven implosion of solid spherical pellets. As a function of power, watts/ $\mathrm{cm}^{2}$ at the initial pellet surface, and critical density, $\rho_{c}$, the figures show a) peak values of $p R, b)$ internal energy in the compressed pellet core at the time of peak $\rho R$, and c) efficiency, $n$, of transfer of ahsorbed laser energy into thc core energy shown in b). 